\title{
The Dispersion Relation for Electrostatic Fluctuations in Weakly Inhomogeneous Plasmas
}

\author{
O. J. G. Silveira ${ }^{1}$, L. F. Ziebell ${ }^{1}$, R. S. Schneider ${ }^{1}$, and R. Gaelzer ${ }^{2}$ \\ ${ }^{1}$ Instituto de Física, UFRGS, Caixa Postal 15051, 91501-970 Porto Alegre, RS, Brasil \\ ${ }^{2}$ Instituto de Física e Matemática, UFPel, Caixa Postal 354, 96010-900 Pelotas, RS, Brasil
}

Received on 21 January, 2004; revised version received on 26 April, 2004

\begin{abstract}
We compare and discuss several approximations to the dispersion relation for electrostatic waves in inhomogeneous plasmas, either obtained directly from Poisson's equation, or from the dielectric constant obtained using a dielectric tensor derived using the plane wave approximation, or from the dielectric constant derived using the effective dielectric tensor.
\end{abstract}

\section{Introduction}

Along the last few years we have conducted several investigations on waves in inhomogeneous plasmas, using the concept of effective dielectric tensor, which has been proposed as the correct dielectric tensor for the description of dielectric properties of inhomogeneous plasmas [1]. Among these investigations, we have considered cases where the magnetic field is homogeneous and other plasma parameters are inhomogeneous $[2,3,4]$, cases where the magnetic field is inhomogeneous and inhomogeneities in the plasma parameters are neglected [5, 6], and cases where inhomogeneities are taken into account both in the plasma parameters and in the magnetic field [7]. For all these cases we case considered arbitrary direction of propagation relative to the ambient magnetic field, and we have taken into account relativistic effects. The expressions obtained for the dielectric tensor for all these cases satisfy Onsager symmetry, and as a consequence the anti-Hermitian part of the tensor only contains resonant terms, as required for proper description of the energy exchange between wave and particles.

We have also applied the concept of effective dielectric tensor to the study of instabilities in the lower hybrid range, in a plasma featuring density and magnetic field inhomogeneities [8]. In the case of this application we have verified that the form of the dispersion relation conventionally used for electromagnetic waves was able to describe the so-called modified two stream instability (MTSI), an instability which occurs due to the existence of a relative drift between ions and electrons [9-13], as well as its purely growing limit for parallel propagation, known as ion Weibel instability (IWI) $[14,15]$. However, the conventional form of the dispersion relation, along with the effective dielectric tensor, was not able to describe the lower hybrid drift instability (LHDI), which is expected to occur when inhomogeneities are taken into account in the description of the electron contribution to the dispersion relation [16-23]. The LHDI is known by its strong electrostatic character, and is frequently studied using the electrostatic approximation. The difficulty of the conventional form of the dispersion relation in describing the LHDI had already been noticed in an earlier analysis, in a formulation which did not use the effective dielectric tensor, adopting instead an ad hoc procedure to correct the lack of symmetry of the dielectric tensor [23]. We have therefore derived a new form of the dispersion relation, taking into account in the derivation the relationship between charge density and electric field expressed by Gauss law, which introduces a term featuring gradients of the inhomogeneous parameters, which is of the same order as other terms due to the inhomogeneity which were taken into account in the derivation of the dielectric tensor. This new form of the dispersion relation was able to describe in a local approximation both the LHDI and the MTSI(IWI), something which was not yet available in the literature up to that moment [8].

The fact that the LHDI was not described with use of the conventional form of the dispersion relation and required a new form of the dispersion relation to take into account all relevant inhomogeneity effects, and the strong electrostatic character of the instability, point out to the relevance of investigating the proper electrostatic limit of the dispersion relation. As it is known, for electrostatic fluctuations in homogeneous plasmas the dispersion relation may be written as $\varepsilon_{l}=0$, where $\varepsilon_{l}$ is the dielectric constant, obtained as follows,

$$
\varepsilon_{l}=\frac{k_{i} \varepsilon_{i j} k_{j}}{k^{2}}
$$

where the $\varepsilon_{i j}$ are the components of the dielectric tensor and the $k_{i}$ are the components of the wave vector $\mathbf{k}$. As a very usual alternative, the dispersion relation for electrostatic waves may also be obtained from Poisson's equation.

When considering the inhomogeneous case, it is necessary to carefully introduce all relevant inhomogeneity effects, and to properly describe the energy exchange between waves and particles. In the present paper we address the subject by comparing and discussing several approximations to the dispersion relation for electrostatic waves in weakly re- 
lativistic inhomogeneous plasmas, either obtained directly from Poisson's equation, or from the dielectric constant obtained using a dielectric tensor derived with the plane wave approximation, or from the dielectric constant derived using the effective dielectric tensor.

\section{The dispersion relation for the elec- trostatic limit}

In the absence of collisions the behavior of the system is ruled by the Vlasov-Maxwell system of equations. Considering small amplitude fluctuations such as the system can be linearized, using the method of characteristics to solve the Vlasov equation, and assuming plane-wave approximation, the perturbed distribution function of a plasma species $\alpha$ can be given by the well known result,

$$
f_{\alpha_{\mathbf{k}, \omega}}=-q_{\alpha} \mathbf{A}_{\alpha} \cdot \mathbf{E}_{\mathbf{k}, \omega}
$$

where

$$
\begin{gathered}
\mathbf{A}_{\alpha} \equiv \int_{-\infty}^{0} \boldsymbol{\Theta}_{\alpha} e^{i(\mathbf{k} \cdot \mathbf{R}-\omega \tau)} d \tau \\
\boldsymbol{\Theta}_{\alpha}=\left(1-\frac{\mathbf{p}^{\prime} \cdot \mathbf{k}}{m_{\alpha} \gamma_{\alpha}^{\prime} \omega}\right) \nabla_{\mathbf{p}^{\prime}} f_{\alpha 0}+\left(\frac{\mathbf{k} \cdot \nabla_{\mathbf{p}^{\prime}} f_{\alpha 0}}{m_{\alpha} \gamma_{\alpha}^{\prime} \omega}\right) \mathbf{p}^{\prime},
\end{gathered}
$$

and

$$
\mathbf{R}=\mathbf{r}^{\prime}-\mathbf{r}, \quad \tau=t^{\prime}-t,
$$

and where Faraday's law has been used to relate magnetic and electric field fluctuations,

$$
\mathbf{B}_{\mathbf{k}, \omega}=\frac{c}{\omega}\left(\mathbf{k} \times \mathbf{E}_{\mathbf{k}, \omega}\right) .
$$

The quantity $m_{\alpha}$ is the mass of the particles of species $\alpha$, $\gamma_{\alpha}$ is the relativistic factor, $\mathbf{p}^{\prime}$ is the unperturbed momentum, and $\mathbf{k}$ and $\omega$ are the wave vector and wave angular frequency, respectively.

The perturbed distribution function is then utilized to obtain the perturbed current. After a considerable amount of algebra, it is well known that a linear relationship, possibly anisotropic, is obtained between the Fourier transforms of current and electric field,

$$
\mathbf{J}_{\mathbf{k}, \omega}=\stackrel{\leftrightarrow}{\sigma}^{0}(\mathbf{k}, \omega ; x) \cdot \mathbf{E}_{\mathbf{k}, \omega}
$$

where

$$
\overleftrightarrow{\sigma}^{0}(\mathbf{k}, \omega ; x)=-\sum_{\alpha} \frac{q_{\alpha}^{2}}{m_{\alpha}} \int d^{3} p \frac{\mathbf{p}}{\gamma_{\alpha}} \mathbf{A}_{\alpha}
$$

The components $\sigma_{i j}^{0}$ appearing in Eq. (4) can be used to define the dielectric tensor,

$$
\stackrel{\leftrightarrow}{\varepsilon}^{0} \equiv \overleftrightarrow{I}+\frac{4 \pi i}{\omega} \stackrel{\leftrightarrow}{\sigma}^{0}
$$

If inhomogeneity effects are explicitly taken into account in the derivation of the $\sigma_{i j}^{0}$ components, there is an inconsistency with the plane wave approximation which is used to describe the fluctuating quantities in the local approximation. As a consequence, the dielectric tensor as given by Eq. (5) features some undesirable properties, like nonresonant terms in the anti-Hermitian part due to the lack of Onsager symmetry of the components $\varepsilon_{i j}^{0}$, and therefore the need to introduce the effective dielectric tensor, aimed to correct the inconsistencies introduced by the local approximation [2].

The calculation of the $\varepsilon_{i j}^{0}$ components for inhomogeneous medium, and the subsequent derivation of the components of the effective dielectric tensor, which we will denote simply as $\varepsilon_{i j}$, can be a very cumbersome task, specially in the case of inhomogeneous magnetic field. In order to pinpoint basic features of the approximation involved in the correct description of the electrostatic limit for inhomogeneous plasmas, we restrict ourselves to the case of homogeneous magnetic field, with inhomogeneities in the parameters appearing in the distribution function assumed to be along the $x$ direction. In what follows we reproduce the expression obtained for the components of the effective dielectric tensor. The details of the calculation can be found elsewhere, although in different notation [2].

$$
\begin{gathered}
\varepsilon_{i j}=\varepsilon_{i j}^{0 h}+\varepsilon_{i j}^{0 n h}+\frac{i}{2} \frac{\partial^{2}}{\partial k_{x} \partial x} \varepsilon_{i j}^{0 h}=\delta_{i j} \\
+\omega \sum_{\alpha} \frac{X_{\alpha}}{n_{\alpha}} \sum_{n=-\infty}^{+\infty} \int d^{3} p p_{\perp} \frac{1}{\gamma_{\alpha} D_{\alpha_{n}}} \pi_{i}^{*} \\
\times\left\{\left(1-\delta_{j z}\right) \varphi_{0}\left(f_{\alpha}\right) \pi_{j}+\delta_{j z}\left[\frac{k_{\perp}}{m_{\alpha} \gamma_{\alpha} \omega} \frac{n}{b_{\alpha}} \mathcal{L}\left(f_{\alpha}\right)+\frac{\partial f_{\alpha}}{\partial p_{\|}}\right] \frac{p_{\perp}}{p_{\|}} \pi_{z}\right\} \\
+\omega \sum_{\alpha} \frac{X_{\alpha}}{n_{\alpha}} \frac{1}{m_{\alpha} \Omega_{\alpha}} \sum_{n=-\infty}^{+\infty} \int d^{3} p p_{\perp}^{2} \frac{1}{\gamma_{\alpha} D_{\alpha_{n}}} \\
\times\left\{\delta_{j y}\left[\left(1-\frac{k_{\|} p_{\|}}{m_{\alpha} \gamma_{\alpha} \omega}\right)-\frac{k_{\perp} p_{\perp}}{m_{\alpha} \gamma_{\alpha} \omega} \frac{n}{b_{\alpha}}\right] \frac{1}{p_{\|}} f_{\alpha}^{\prime} \pi_{i}^{*} \pi_{z}\right. \\
+\frac{k_{\perp} \sin \psi}{m_{\alpha} \gamma_{\alpha} \omega} f_{\alpha}^{\prime} \pi_{i}^{*} \pi_{j}+\left(1-\delta_{j z}\right) \varphi_{0}\left(f_{\alpha}^{\prime}\right)\left(\Phi_{i}^{*} \pi_{j}\right)^{H} \\
\left.+\delta_{j z}\left[\frac{k_{\perp}}{m_{\alpha} \gamma_{\alpha} \omega} \frac{n}{b_{\alpha}} \mathcal{L}\left(f_{\alpha}^{\prime}\right)+\frac{\partial f_{\alpha}^{\prime}}{\partial p_{\|}}\right] \frac{p_{\perp}}{p_{\|}}\left(\Phi_{i}^{*} \pi_{z}\right)^{H}\right\}
\end{gathered}
$$

where

$$
\begin{aligned}
\mathcal{L} & =p_{\|} \frac{\partial}{\partial p_{\perp}}-p_{\perp} \frac{\partial}{\partial p_{\|}}, \\
\varphi_{0} & =\frac{\partial}{\partial p_{\perp}}-\frac{k_{\|}}{m_{\alpha} \gamma_{\alpha} \omega} \mathcal{L}, \\
D_{\alpha_{n}} & =\omega-\frac{n \Omega_{\alpha}}{\gamma_{\alpha}}-\frac{k_{\|} p_{\|}}{m_{\alpha} \gamma_{\alpha}} .
\end{aligned}
$$

The $\pi_{i}$ and $\Phi_{i}$ appearing in Eq. (6) are the components of the following vectors,

$$
\begin{aligned}
\vec{\pi} & =\left(\frac{n J_{n}\left(b_{\alpha}\right)}{b_{\alpha}} \cos \psi-i J_{n}^{\prime}\left(b_{\alpha}\right) \sin \psi\right) \mathbf{e}_{\mathbf{x}} \\
& +\left(\frac{n J_{n}\left(b_{\alpha}\right)}{b_{\alpha}} \sin \psi+i J_{n}^{\prime}\left(b_{\alpha}\right) \cos \psi\right) \mathbf{e}_{\mathbf{y}}
\end{aligned}
$$




$$
\begin{gathered}
+\frac{p_{\|}}{p_{\perp}} J_{n}\left(b_{\alpha}\right) \mathbf{e}_{\mathbf{z}} \\
\vec{\Phi}=\left\{\left[\left(\frac{n^{2}}{b_{\alpha}^{2}}-\frac{1}{2}\right) J_{n}\left(b_{\alpha}\right)-\frac{J_{n}^{\prime}\left(b_{\alpha}\right)}{b_{\alpha}}\right] \sin (2 \psi)\right. \\
\left.-i\left[\frac{n}{b_{\alpha}^{2}} J_{n}\left(b_{\alpha}\right)-\frac{n J_{n}^{\prime}\left(b_{\alpha}\right)}{b_{\alpha}}\right] \cos (2 \psi)\right\} \mathbf{e}_{\mathbf{x}} \\
+\left\{\frac{J_{n}}{2}-\left[\left(\frac{n^{2}}{b_{\alpha}^{2}}-\frac{1}{2}\right) J_{n}\left(b_{\alpha}\right)-\frac{J_{n}^{\prime}\left(b_{\alpha}\right)}{b_{\alpha}}\right] \cos (2 \psi)\right. \\
\left.-i\left[\frac{n}{b_{\alpha}^{2}} J_{n}\left(b_{\alpha}\right)-\frac{n J_{n}^{\prime}\left(b_{\alpha}\right)}{b_{\alpha}}\right] \sin (2 \psi)\right\} \mathbf{e}_{\mathbf{y}} \\
+\frac{p_{\|}}{p_{\perp}}\left[\frac{n}{b_{\alpha}} J_{n}\left(b_{\alpha}\right) \sin \psi+i J_{n}^{\prime}\left(b_{\alpha}\right) \cos \psi\right] \mathbf{e}_{\mathbf{z}},
\end{gathered}
$$

where $b_{\alpha}=k_{\perp} p_{\perp} /\left(m_{\alpha} \Omega_{\alpha}\right)$, with $\Omega_{\alpha}$ being the cyclotron angular frequency of particles of species $\alpha$. The quantity $X_{\alpha}$ appearing in Eq. (6) is defined as $\omega_{\alpha}^{2} / \omega^{2}$, where $\omega_{\alpha}$ is the plasma angular frequency of particles of species $\alpha$, and $n_{\alpha}$ is the density of these particles. The components of the wave vector respectively perpendicular and parallel to the direction of the ambient magnetic field are $k_{\perp}$ and $k_{\|}$, with $\psi$ being the angle between $\mathbf{k}_{\perp}$ and the direction of the inhomogeneity.

We notice in Eq. (6) the presence of the quotient between the quantity $k_{\perp}$, which appears due to the magnetic fluctuations introduced by Faraday's law, and the quantity $b_{\alpha}$ which appears in the denominator. Since the quantity $b_{\alpha}$ is proportional to $k_{\perp}$, its definition can be used to cancel out the $k_{\perp}$ appearing in the numerator. The outcome is a different form for the effective dielectric tensor.

$$
\begin{aligned}
& \varepsilon_{i j}=\delta_{i j}+\omega \sum_{\alpha} \frac{X_{\alpha}}{n_{\alpha}} \sum_{n=-\infty}^{+\infty} \int d^{3} p p_{\perp} \frac{1}{\gamma_{\alpha} D_{\alpha_{n}}} \varphi_{0}\left(f_{\alpha}\right) \pi_{i}^{*} \pi_{j} \\
&-\delta_{i z} \delta_{j z} \sum_{\alpha} \frac{X_{\alpha}}{n_{\alpha}} \int d^{3} p \frac{1}{\gamma_{\alpha}} \frac{p_{\|}}{p_{\perp}} \mathcal{L}\left(f_{\alpha}\right) \\
&+ \delta_{j y} \delta_{i z} \sum_{\alpha} \frac{X_{\alpha}}{n_{\alpha}} \frac{1}{m_{\alpha} \Omega_{\alpha}} \int d^{3} p p_{\perp} \frac{1}{\gamma_{\alpha}} \frac{p_{\|}}{p_{\perp}} f_{\alpha}^{\prime} \\
&+ \omega \sum_{\alpha} \frac{X_{\alpha}}{n_{\alpha}} \frac{1}{m_{\alpha} \Omega_{\alpha}} \sum_{n=-\infty}^{+\infty} \int d^{3} p p_{\perp}^{2} \frac{1}{\gamma_{\alpha} D_{\alpha_{n}}} \\
& \times\left[\frac{k_{\perp}}{m_{\alpha} \gamma_{\alpha} \omega} f_{\alpha}^{\prime} \pi_{i}^{*} \pi_{j}+\varphi_{0}\left(f_{\alpha}^{\prime}\right)\left(\Phi_{i}^{*} \pi_{j}\right)^{H}\right] \\
&-\delta_{j z} \sum_{\alpha} \frac{X_{\alpha}}{n_{\alpha}} \frac{1}{m_{\alpha} \Omega_{\alpha}} \sum_{n=-\infty}^{+\infty} \int d^{3} p p_{\perp} \frac{1}{\gamma_{\alpha}} \frac{p_{\perp}}{p_{\|}} \mathcal{L}\left(f_{\alpha}^{\prime}\right)\left(\Phi_{i}^{*} \pi_{j}\right)^{H} .
\end{aligned}
$$

We examine now the last term in this expression. Since there is no resonant denominator, the following property can be used,

$$
\sum_{n=-\infty}^{+\infty}\left(\Phi_{i}^{*} \pi_{z}\right)^{H}=\sum_{n=-\infty}^{+\infty}\left(\frac{p_{\|}}{p_{\perp}}\right)^{\delta_{i z}+1} S_{i z}^{H}=\frac{1}{2} \frac{p_{\|}}{p_{\perp}} \delta_{i y}
$$

where the definition of $S_{i z}$ can be found in Ref. [2], and therefore the term with the $\delta_{j z}$ in Eq. (9) can be written as

$$
\begin{gathered}
-\delta_{i y} \delta_{j z} \frac{1}{2} \sum_{\alpha} \frac{X_{\alpha}}{n_{\alpha}} \frac{1}{m_{\alpha} \Omega_{\alpha}} \int d^{3} p p_{\perp} \frac{1}{\gamma_{\alpha}} \mathcal{L}\left(f_{\alpha}^{\prime}\right) \\
=\delta_{i y} \delta_{j z} \sum_{\alpha} \frac{X_{\alpha}}{n_{\alpha}} \frac{1}{m_{\alpha} \Omega_{\alpha}} \int d^{3} p \frac{1}{\gamma_{\alpha}} p_{\|} f_{\alpha}^{\prime},
\end{gathered}
$$

where the last step was obtained via integration by parts.

Using this result in Eq. (9),

$$
\begin{aligned}
\varepsilon_{i j}=\delta_{i j} & +\omega \sum_{\alpha} \frac{X_{\alpha}}{n_{\alpha}} \sum_{n=-\infty}^{+\infty} \int d^{3} p p_{\perp} \frac{1}{\gamma_{\alpha} D_{\alpha_{n}}} \varphi_{0}\left(f_{\alpha}\right) \pi_{i}^{*} \pi_{j} \\
& -\delta_{i z} \delta_{j z} \sum_{\alpha} \frac{X_{\alpha}}{n_{\alpha}} \int d^{3} p \frac{1}{\gamma_{\alpha}} \frac{p_{\|}}{p_{\perp}} \mathcal{L}\left(f_{\alpha}\right) \\
+ & \omega \sum_{\alpha} \frac{X_{\alpha}}{n_{\alpha}} \frac{1}{m_{\alpha} \Omega_{\alpha}} \sum_{n=-\infty}^{+\infty} \int d^{3} p p_{\perp}^{2} \frac{1}{\gamma_{\alpha} D_{\alpha_{n}}} \\
\times & {\left[\frac{k_{\perp} \sin \psi}{m_{\alpha} \gamma_{\alpha} \omega} f_{\alpha}^{\prime} \pi_{i}^{*} \pi_{j}+\varphi_{0}\left(f_{\alpha}^{\prime}\right)\left(\Phi_{i}^{*} \pi_{j}\right)^{H}\right] } \\
+\left(\delta_{i y} \delta_{j z}\right. & \left.+\delta_{j y} \delta_{i z}\right) \sum_{\alpha} \frac{X_{\alpha}}{n_{\alpha}} \frac{1}{m_{\alpha} \Omega_{\alpha}} \int d^{3} p p_{\perp} \frac{1}{\gamma_{\alpha}} \frac{p_{\|}}{p_{\perp}} f_{\alpha}^{\prime}
\end{aligned}
$$

These expressions for the components of the effective dielectric tensor are exactly equivalent to those obtained in Ref. [2].

Once we have the proper dielectric tensor, let us examine the dispersion relation. In the electrostatic approximation, $\mathbf{B}_{\mathbf{1}} \simeq 0$, and Gauss's law can be used to obtain the dispersion relation. Using it along with continuity's equation, and using plane wave approximation for the electric field, we obtain the following

$$
i \mathbf{k} \cdot \mathbf{E}=-\frac{4 \pi i}{\omega}[(\nabla \cdot \overleftrightarrow{\sigma}) \cdot \mathbf{E}+i \mathbf{k} \cdot \overleftrightarrow{\sigma} \cdot \mathbf{E}]
$$

where we have used the relationship between the effective dielectric tensor and the effective conductivity,

$$
\varepsilon_{i j}=\delta_{i j}+\frac{4 \pi i}{\omega} \sigma_{i j} .
$$

Using $\mathbf{E}=-\nabla \phi=-i \mathbf{k} \phi$, we obtain the following dispersion relation,

$$
k^{2} \varepsilon_{l}-i \mathbf{k} \cdot(\nabla \cdot \overleftrightarrow{\varepsilon})=0 .
$$

The general form of this dispersion relation can be found in well known textbooks [24]. However, we point out that the dielectric tensor to be used in Eq. (12) is the effective dielectric tensor, which is free from the inconsistencies arising from the use of the local approximation for inhomogeneous plasmas. Using the effective dielectric tensor as given by Eq. (6), we obtain

$$
\varepsilon_{l}=1+\frac{\omega^{2}}{k^{2}} \sum_{\alpha} \frac{X_{\alpha}}{n_{\alpha}} m_{\alpha} \sum_{n=-\infty}^{+\infty} \int d^{3} p \frac{1}{D_{\alpha_{n}}} J_{n}^{2}
$$




$$
\begin{gathered}
\times\left[k_{\perp} \frac{n}{b_{\alpha}} \frac{\partial f_{\alpha}}{\partial p_{\perp}}+k_{\|} \frac{\partial f_{\alpha}}{\partial p_{\|}}\right] \\
+\frac{\omega^{2}}{k^{2}} \sum_{\alpha} \frac{X_{\alpha}}{n_{\alpha}} \frac{k_{\perp} \sin \psi}{\Omega_{\alpha}} \sum_{n=-\infty}^{+\infty} \int d^{3} p \frac{1}{D_{\alpha_{n}}} J_{n}^{2} f_{\alpha}^{\prime} \\
+\frac{\omega^{2}}{k^{2}} \sum_{\alpha} \frac{X_{\alpha}}{n_{\alpha}} \frac{\sin \psi}{\Omega_{\alpha}} \sum_{n=-\infty}^{+\infty} \int d^{3} p p_{\perp} \frac{1}{D_{\alpha_{n}}} \\
\times\left[\frac{n J_{n}^{2}}{b_{\alpha}}-\frac{\Omega_{\alpha}}{\gamma_{\alpha} \omega} J_{n} J_{n}^{\prime}\right]\left[k_{\perp} \frac{n}{b_{\alpha}} \frac{\partial f_{\alpha}^{\prime}}{\partial p_{\perp}}+k_{\|} \frac{\partial f_{\alpha}^{\prime}}{\partial p_{\|}}\right] .
\end{gathered}
$$

Details about this calculation can be found in Appendix A. It is important to notice that all the $k_{i}$ components originated from the magnetic fluctuations have been cancelled out in the derivation of Eq. (13), so that the dielectric constant is the same as it would be obtained if we had started assuming electrostatic fluctuations when deriving the components of the dielectric tensor.

By taking into account that the inhomogeneities are along the $x$ direction, evaluating the derivatives of the components of the dielectric tensor as given by Eq. (6), and using Eq. (13), the dispersion relation (12) can be written as follows,

$$
\begin{gathered}
-i \frac{\omega}{k^{2}} \sum_{\alpha} \frac{X_{\alpha}}{n_{\alpha}} \sum_{n=-\infty}^{+\infty} \int d^{3} p p_{\perp} \frac{1}{\gamma_{\alpha} D_{\alpha_{n}}}\left[k_{\perp} \frac{n}{b_{\alpha}} \frac{\partial f_{\alpha}^{\prime}}{\partial p_{\perp}}+k_{\|} \frac{\partial f_{\alpha}^{\prime}}{\partial p_{\|}}\right] \\
\times\left(\frac{n J_{n}^{2}}{b_{\alpha}} \cos \psi+i J_{n} J_{n}^{\prime} \sin \psi\right)+\varepsilon_{l}=0
\end{gathered}
$$

\section{Other approximations to the dis- persion relation}

As we have pointed out, the so-called BGI procedure has played an essential role in correcting inconsistencies arising from the use of a plane wave approximation in an inhomogeneous plasma. However, it may be illuminating to compare the dispersion relation obtained using the effective dielectric tensor, Eq. (14), with the corresponding dispersion relation obtained using the dielectric tensor derived with use of the plane wave approximation, with components $\varepsilon_{i j}^{0}$. The components of this tensor can be given as follows,

$$
\begin{gathered}
\varepsilon_{i j}^{0}=\delta_{i j}+\omega \sum_{\alpha} \frac{X_{\alpha}}{n_{\alpha}} \sum_{n=-\infty}^{+\infty} \int d^{3} p p_{\perp} \frac{1}{\gamma_{\alpha} D_{\alpha n}} \\
\times\left\{\left(1-\delta_{j z}\right)\left[\frac{\partial f_{\alpha}}{\partial p_{\perp}}-\frac{k_{\|}}{m_{\alpha} \gamma_{\alpha} \omega} \mathcal{L}\left(f_{\alpha}\right)\right] \pi_{i}^{*} \pi_{j}\right. \\
\left.+\delta_{j z}\left[\frac{k_{\perp}}{m_{\alpha} \gamma_{\alpha} \omega} \frac{n}{b_{\alpha}} \mathcal{L}\left(f_{\alpha}\right)+\frac{\partial f_{\alpha}}{\partial p_{\|}}\right] \frac{p_{\perp}}{p_{\|}} \pi_{i}^{*} \pi_{z}\right\} \\
+\omega \sum_{\alpha} \frac{X_{\alpha}}{n_{\alpha}} \frac{1}{m_{\alpha} \Omega_{\alpha}} \sum_{n=-\infty}^{+\infty} \int d^{3} p p_{\perp}^{2} \frac{1}{\gamma_{\alpha} D_{\alpha n}} \\
\times\left\{\delta_{j y}\left[1-\frac{k_{\|} p_{\|}}{m_{\alpha} \gamma_{\alpha} \omega}-\frac{k_{\perp} p_{\perp}}{m_{\alpha} \gamma_{\alpha} \omega} \frac{n}{b_{\alpha}}\right] \frac{1}{p_{\|}} f_{\alpha}^{\prime} \pi_{i}^{*} \pi_{z}\right.
\end{gathered}
$$

$$
\begin{gathered}
+\frac{k_{\perp} \sin \psi}{m_{\alpha} \gamma_{\alpha} \omega} f_{\alpha}^{\prime} \pi_{i}^{*} \pi_{j}+\left(1-\delta_{j z}\right)\left[\frac{\partial f_{\alpha}^{\prime}}{\partial p_{\perp}}-\frac{k_{\|}}{m_{\alpha} \gamma_{\alpha} \omega} \mathcal{L}\left(f_{\alpha}^{\prime}\right)\right] \Phi_{i}^{*} \pi_{j} \\
\left.+\delta_{j z}\left[\frac{k_{\perp}}{m_{\alpha} \gamma_{\alpha} \omega} \frac{n}{b_{\alpha}} \mathcal{L}\left(f_{\alpha}^{\prime}\right)+\frac{\partial f_{\alpha}^{\prime}}{\partial p_{\|}}\right] \frac{p_{\perp}}{p_{\|}} \Phi_{i}^{*} \pi_{z}\right\}
\end{gathered}
$$

This expression is the counterpart of Eq. (6), prior the BGI procedure. Using the $\varepsilon_{i j}^{0}$ components with Eq. (1), and following procedures similar to those utilized in Appendix A to derive the effective dielectric constant, we obtain a dielectric constant which do not describe adequately the energy exchange between waves and particles, and which will be denoted as $\varepsilon_{l}^{0}$,

$$
\begin{gathered}
\varepsilon_{l}^{0}=1+\frac{\omega}{k^{2}} \sum_{\alpha} \frac{X_{\alpha}}{n_{\alpha}} \sum_{n=-\infty}^{+\infty} \int d^{3} p p_{\perp} \frac{1}{\gamma_{\alpha} D_{\alpha n}} J_{n}^{2} \\
\times\left(k_{\perp} \frac{n}{b_{\alpha}}+k_{\|} \frac{p_{\|}}{p_{\perp}}\right)\left(k_{\perp} \frac{n}{b_{\alpha}} \frac{\partial f_{\alpha}}{\partial p_{\perp}}+k_{\|} \frac{\partial f_{\alpha}}{\partial p_{\|}}\right) \\
+\frac{\omega}{k^{2}} \sum_{\alpha} \frac{X_{\alpha}}{n_{\alpha}} \frac{1}{m_{\alpha} \Omega_{\alpha}} \sum_{n=-\infty}^{+\infty} \int d^{3} p p_{\perp}^{2} \frac{1}{\gamma_{\alpha} D_{\alpha n}} \\
\times\left\{\frac{1}{p_{\perp}} f_{\alpha}^{\prime} J_{n}^{2} k_{\perp} \sin \psi\left(k_{\perp} \frac{n}{b_{\alpha}}+k_{\|} \frac{p_{\|}}{p_{\perp}}\right)\right. \\
+\left[k_{\perp} \sin \psi\left(\frac{n^{2} J_{n}^{2}}{b_{\alpha}^{2}}-\frac{J_{n} J_{n}^{\prime}}{b_{\alpha}}\right)+i k_{\perp} \cos \psi\left(\frac{n J_{n}^{2}}{b_{\alpha}^{2}}-\frac{n J_{n} J_{n}^{\prime}}{b_{\alpha}}\right)\right. \\
\left.+k_{\|} \frac{p_{\|}}{p_{\perp}}\left(\frac{n J_{n}^{2}}{b_{\alpha}} \sin \psi-i J_{n} J_{n}^{\prime} \cos \psi\right)\right] \\
\left.\times\left(k_{\perp} \frac{n}{b_{\alpha}} \frac{\partial f_{\alpha}^{\prime}}{\partial p_{\perp}}+k_{\|} \frac{\partial f_{\alpha}^{\prime}}{\partial p_{\|}}\right)\right\} .
\end{gathered}
$$

By comparing Eqs. (13) and (16) it is easy to show the following result

$$
\begin{gathered}
\varepsilon_{l}-\varepsilon_{l}^{0}=-i \frac{\omega^{2}}{k^{2}} \cos \psi \sum_{\alpha} \frac{X_{\alpha}}{n_{\alpha}} \frac{1}{\Omega_{\alpha}} \sum_{n=-\infty}^{+\infty} \int d^{3} p p_{\perp} \frac{1}{D_{\alpha n}} \\
\times\left[\frac{\Omega_{\alpha}}{\gamma_{\alpha} \omega} \frac{n J_{n}^{2}}{b_{\alpha}}-J_{n} J_{n}^{\prime}\right]\left(k_{\perp} \frac{n}{b_{\alpha}} \frac{\partial f_{\alpha}^{\prime}}{\partial p_{\perp}}+k_{\|} \frac{\partial f_{\alpha}^{\prime}}{\partial p_{\|}}\right) \cdot
\end{gathered}
$$

Eq. (17) shows that the dielectric constants originated from the effective and from the plane wave tensors are in general different, even in the simple case considered here, of homogeneous magnetic field and gradients of plasma parameters pointing along a direction perpendicular to the magnetic field. These two forms of the dielectric constant are clearly equal only in the case of waves propagating perpendicularly to the direction of the inhomogeneity. This conclusion can also be extended to the dispersion relation itself, since it is trivial to show that the dispersion relation using plane wave approximation would be the same as Eq. (14) with $\varepsilon_{l}^{0}$ replacing $\varepsilon_{l}$. Of course, this dispersion relation featuring the plane wave tensor instead of the effective dielectric tensor is not appropriate to describe the electrostatic oscillations, since it would not describe properly the energy exchange between waves and particles. 
Another approach to the dispersion relation is largely used in the treatment of electrostatic waves in inhomogeneous media, and can be summarized as follows. Starting from Gauss's law, using the electrostatic approximation $\mathbf{E}_{1}=-\nabla \phi_{1}$, and assuming plane wave approximation, we obtain

$$
k^{2} \phi=4 \pi \sum_{\alpha} q_{\alpha} \int d^{3} p f_{\alpha},
$$

where the (Fourier-transformed) distribution function can be obtained from Vlasov equation according to Eq. (2). Following procedures similar to those employed to derive the $\varepsilon_{i j}^{0}$ components, after some reasonable amount of algebraic manipulation we obtain the relationship between charge density and fluctuating electric field, and therefore obtain the dispersion relation as follows,

$$
\begin{gathered}
\varepsilon_{l}^{P}=1+\frac{\omega^{2}}{k^{2}} \sum_{\alpha} \frac{X_{\alpha} m_{\alpha}}{n_{\alpha}} \sum_{n=-\infty}^{+\infty} \int d^{3} p \frac{1}{D_{\alpha n}} J_{n}^{2} \\
\times\left(k_{\perp} \frac{n}{b_{\alpha}} \frac{\partial f_{\alpha}}{\partial p_{\perp}}+k_{\|} \frac{\partial f_{\alpha}}{\partial p_{\|}}\right) \\
+\frac{\omega^{2}}{k^{2}} \sin \psi \sum_{\alpha} \frac{X_{\alpha}}{n_{\alpha} \Omega_{\alpha}} \sum_{n=-\infty}^{+\infty} \int d^{3} p p_{\perp} \frac{1}{D_{\alpha n}} J_{n}^{2} \\
\times\left[\frac{n}{b_{\alpha}}\left(k_{\perp} \frac{n}{b_{\alpha}} \frac{\partial f_{\alpha}^{\prime}}{\partial p_{\perp}}+k_{\|} \frac{\partial f_{\alpha}^{\prime}}{\partial p_{\|}}\right)+k_{\perp} \frac{1}{p_{\perp}} f_{\alpha}^{\prime}\right] \\
-i \cos \psi \frac{\omega^{2}}{k^{2}} \sum_{\alpha} \frac{X_{\alpha}}{n_{\alpha} \Omega_{\alpha}} \sum_{n=-\infty}^{+\infty} \int d^{3} p p_{\perp} \frac{1}{D_{\alpha n}} J_{n} J_{n}^{\prime} \\
\times\left(k_{\perp} \frac{n}{b_{\alpha}} \frac{\partial f_{\alpha}^{\prime}}{\partial p_{\perp}}+k_{\|} \frac{\partial f_{\alpha}^{\prime}}{\partial p_{\|}}\right)=0 .
\end{gathered}
$$

It is important to remark that in this approach inhomogeneity effects have been included in the dispersion relation along with a plane wave approximation to describe the fluctuating quantities. As we know, this approximation generates inconsistencies. However, in the derivation of Eq. (18) no correction along the lines of the BGI procedure employed to derive the effective dielectric tensor has been applied, and therefore the dispersion relation as given by Eq. (18) may not describe adequately the energy exchange between waves and particles, as it is also the case of the dispersion relation obtained using the $\varepsilon_{i j}^{0}$ components.

By comparing the dielectric constant Eq. (18) obtained with use of Poisson's equation with the effective dielectric constant given by Eq. (13), we obtain the following

$$
\begin{aligned}
\varepsilon_{l}= & \varepsilon_{l}^{P}-\frac{\omega}{k^{2}} \sin \psi \sum_{\alpha} \frac{X_{\alpha}}{n_{\alpha}} \sum_{n=-\infty}^{+\infty} \int d^{3} p p_{\perp} \frac{1}{\gamma_{\alpha} D_{\alpha_{n}}} J_{n} J_{n}^{\prime} \\
& \times\left[k_{\perp} \frac{n}{b_{\alpha}} \frac{\partial f_{\alpha}^{\prime}}{\partial p_{\perp}}+k_{\|} \frac{\partial f_{\alpha}^{\prime}}{\partial p_{\|}}\right] \\
& +i \cos \psi \frac{\omega^{2}}{k^{2}} \sum_{\alpha} \frac{X_{\alpha}}{n_{\alpha} \Omega_{\alpha}} \sum_{n=-\infty}^{+\infty} \int d^{3} p p_{\perp} \frac{1}{D_{\alpha n}} J_{n} J_{n}^{\prime}
\end{aligned}
$$

$$
\times\left[k_{\perp} \frac{n}{b_{\alpha}} \frac{\partial f_{\alpha}^{\prime}}{\partial p_{\perp}}+k_{\|} \frac{\partial f_{\alpha}^{\prime}}{\partial p_{\|}}\right] .
$$

Using this expression along with the dispersion relation given by Eq. (14), it is seen that the dispersion relation may be written as follows,

$$
\begin{gathered}
\varepsilon_{l}^{P}-i \frac{\omega}{k^{2}} \cos \psi \sum_{\alpha} \frac{X_{\alpha}}{n_{\alpha}} \sum_{n=-\infty}^{+\infty} \int d^{3} p p_{\perp} \frac{1}{\gamma_{\alpha} D_{\alpha_{n}}} \\
\times\left[k_{\perp} \frac{n}{b_{\alpha}} \frac{\partial f_{\alpha}^{\prime}}{\partial p_{\perp}}+k_{\|} \frac{\partial f_{\alpha}^{\prime}}{\partial p_{\|}}\right]\left(\frac{n J_{n}^{2}}{b_{\alpha}}-J_{n} J_{n}^{\prime}\right)=0 .
\end{gathered}
$$

From this expression, we conclude that for propagation perpendicular to the inhomogeneity $(\psi=\pi / 2)$, the dispersion relation obtained from Poisson's equation, without BGI correction, is exactly the same as the correct dispersion relation which has incorporated all the relevant inhomogeneity effects and which has been corrected using the BGI procedure, namely Eq. (14). For general directions of propagation, however, these two forms of the dispersion relation do not in general coincide.

\section{Conclusions}

We have investigated the electrostatic limit of the dispersion relation for weakly inhomogeneous media, by comparing and discussing several approximations to the dispersion relation. We have started by deriving the dispersion relation using Gauss's law, taking into account the equation of charge continuity to relate charge and current densities, and expressing the relationship between current density and electric field by means of the effective conductivity, which is derived using the so called BGI procedure in order to guarantee proper description of the energy exchange between waves and particles. We have seen that another form of the dispersion relation derived with a conductivity tensor which has not been corrected using the BGI procedure in general does not correspond to the correct form of the dispersion relation, except for propagation perpendicular to the direction of inhomogeneity. This result is not surprising, since it is known that for this particular direction the uncorrected tensor corresponds to the effective conductivity tensor.

We have also derived the dispersion relation using Poisson's equation and direct evaluation of charge density, taking into account inhomogeneity effects. For propagation perpendicular to the direction of inhomogeneity, the equation obtained following this procedure is the same as the correct equation derived using the effective dielectric tensor. For general directions of propagation, however, the equation obtained differs from the correct dispersion relation, which is not surprising, since in the derivation starting from Poisson's equation no BGI correction has been applied, and therefore there is no guarantee of correct description of the wave-particle interaction. 


\section{A The derivation of the effective die- lectric constant}

Using Eq. (1) with the components of the effective dielectric tensor as given by Eq. (6), we use the following results,

$$
\begin{gathered}
\sum_{i j} k_{i} \delta_{i j} k_{j}=\sum_{i} k_{i} k_{i}=k^{2}, \\
\sum_{i j} k_{i} \delta_{i z} \delta_{j z} k_{j}=k_{z} k_{z}=k_{\|}^{2}, \\
\sum_{i j} k_{i}\left(\delta_{i y} \delta_{j z}+\delta_{j y} \delta_{i z}\right) k_{j}=2 k_{y} k_{z} \\
=2 k_{\|} k_{\perp} \sin \psi,
\end{gathered}
$$

and obtain after some algebraic manipulations,

$$
\begin{gathered}
\varepsilon_{l}=1+\frac{\omega}{k^{2}} \sum_{\alpha} \frac{X_{\alpha}}{n_{\alpha}} \sum_{n=-\infty}^{+\infty} \int d^{3} p p_{\perp} \frac{1}{\gamma_{\alpha} D_{\alpha_{n}}} \\
\times\left\{\left(\frac{\partial f_{\alpha}}{\partial p_{\perp}}-\frac{k_{\|}}{m_{\alpha} \gamma_{\alpha} \omega} \mathcal{L}\left(f_{\alpha}\right)\right) \sum_{i j} k_{i} \pi_{i}^{*} \pi_{j} k_{j}\right. \\
\left.+\frac{1}{p_{\|}} \mathcal{L}\left(f_{\alpha}\right) \sum_{i} k_{i} \pi_{i}^{*} \pi_{z} k_{\|}\left[-1+\frac{k_{\|} p_{\|}}{m_{\alpha} \gamma_{\alpha} \omega}+\frac{k_{\perp} p_{\perp}}{m_{\alpha} \gamma_{\alpha} \omega} \frac{n}{b_{\alpha}}\right]\right\} \\
+\frac{\omega}{k^{2}} \sum_{\alpha} \frac{X_{\alpha}}{n_{\alpha}} \frac{1}{m_{\alpha} \Omega_{\alpha}} \sum_{n=-\infty}^{+\infty} \int d^{3} p p_{\perp}^{2} \frac{1}{\gamma_{\alpha} D_{\alpha_{n}}} \\
\times\left\{\left[\begin{array}{l}
k_{\|} p_{\|} \\
m_{\alpha} \gamma_{\alpha} \omega
\end{array}-\frac{k_{\perp} p_{\perp}}{m_{\alpha} \gamma_{\alpha} \omega} \frac{n}{b_{\alpha}}\right] \frac{1}{p_{\|}} k_{\perp} \sin \psi f_{\alpha}^{\prime} \sum_{i} k_{i} \pi_{i}^{*} \pi_{z}\right. \\
+\frac{k_{\perp} \sin \psi}{m_{\alpha} \gamma_{\alpha} \omega} f_{\alpha}^{\prime} \sum_{i j} \mathcal{L}\left(f_{\alpha}^{\prime}\right) \sum_{i} k_{i}^{*} \pi_{j} k_{j} \\
+\left(\frac{\partial f_{\alpha}^{\prime}}{\partial p_{\perp}}-\frac{k_{\|}}{m_{\alpha} \gamma_{\alpha} \omega} \mathcal{L}\left(f_{\alpha}^{\prime}\right)\right) \sum_{i j} k_{i}\left(\Phi_{i}^{*} \pi_{j}\right)^{H} k_{j} \\
\left.k_{\|}\left[-1+\frac{k_{\|} p_{\|}}{m_{\alpha} \gamma_{\alpha} \omega}+\frac{k_{\perp} p_{\perp}}{m_{\alpha} \gamma_{\alpha} \omega} \frac{n}{b_{\alpha}}\right]\right\}
\end{gathered}
$$

Using the definition of the $\pi_{i}$, we obtain

$$
\begin{gathered}
\sum_{i} k_{i} \pi_{i}=J_{n}\left(\frac{n}{b_{\alpha}} k_{\perp}+k_{\|} \frac{p_{\|}}{p_{\perp}}\right) \\
\sum_{i j} k_{i} \pi_{i}^{*} \pi_{j} k_{j}=J_{n}^{2}\left(\frac{n}{b_{\alpha}} k_{\perp}+k_{\|} \frac{p_{\|}}{p_{\perp}}\right)^{2}
\end{gathered}
$$

We also need the following quantity,

$$
\sum_{i j} k_{i}\left(\Phi_{i}^{*} \pi_{j}\right)^{H} k_{j}=\frac{1}{2}\left[\left(\mathbf{k} \cdot \vec{\Phi}^{*}\right)(\mathbf{k} \cdot \vec{\pi})+\text { c.c. }\right] \text {. }
$$

Since the quantity $k_{i} \pi_{i}$ is real,

$$
\frac{1}{2}\left[\left(\mathbf{k} \cdot \vec{\Phi}^{*}\right)(\mathbf{k} \cdot \vec{\pi})+\text { c.c. }\right]=(\mathbf{k} \cdot \vec{\pi})(\mathbf{k} \cdot \operatorname{Re} \vec{\Phi}) .
$$

Using the definition of the $\Phi_{i}$,

$$
\begin{gathered}
\sum_{i} k_{i} \operatorname{Re} \Phi_{i}=k_{\perp}\left[\frac{n^{2} J_{n}}{b_{\alpha}^{2}}-\frac{J_{n}}{2}-\frac{J_{n}^{\prime}}{b_{\alpha}}\right] \sin (2 \psi) \cos \psi \\
+k_{\perp}\left\{\frac{J_{n}}{2}-\left[\frac{n^{2} J_{n}}{b_{\alpha}^{2}}-\frac{J_{n}}{2}-\frac{J_{n}^{\prime}}{b_{\alpha}}\right] \cos (2 \psi)\right\} \sin \psi \\
+k_{\|} \frac{p_{\|}}{p_{\perp}} \frac{n}{b_{\alpha}} J_{n} \sin \psi \\
=\operatorname{Re} \Phi_{z}^{*}\left(k_{\perp} \frac{p_{\perp}}{p_{\|}} \frac{n}{b_{\alpha}}+k_{\|}\right)-k_{\perp} \frac{J_{n}^{\prime}}{b_{\alpha}} \sin \psi
\end{gathered}
$$

Therefore,

$$
\begin{gathered}
\sum_{i j} k_{i}\left(\Phi_{i}^{*} \pi_{j}\right)^{H} k_{j}=\left(\sum_{i} k_{i} \pi_{i}\right)\left(\sum_{i} k_{i} \operatorname{Re} \Phi_{i}\right) \\
=\left(k_{\perp} \frac{p_{\perp}}{p_{\|}} \frac{n}{b_{\alpha}}+k_{\|}\right) \pi_{z} \sin \psi \\
\times\left[\frac{n}{b_{\alpha}} \pi_{z}\left(k_{\perp} \frac{p_{\perp}}{p_{\|}} \frac{n}{b_{\alpha}}+k_{\|}\right)-k_{\perp} \frac{J_{n}^{\prime}}{b_{\alpha}}\right] .
\end{gathered}
$$

Using Eqs. (26) and (27) in Eq. (24),

$$
\begin{aligned}
& \varepsilon_{l}=1+\frac{\omega}{k^{2}} \sum_{\alpha} \frac{X_{\alpha}}{n_{\alpha}} \sum_{n=-\infty}^{+\infty} \int d^{3} p p_{\perp} \frac{1}{\gamma_{\alpha} D_{\alpha_{n}}} J_{n}^{2}\left(\frac{n}{b_{\alpha}} k_{\perp}+k_{\|} \frac{p_{\|}}{p_{\perp}}\right) \\
& \times\left\{\left(\frac{\partial f_{\alpha}}{\partial p_{\perp}}-\frac{k_{\|}}{m_{\alpha} \gamma_{\alpha} \omega} \mathcal{L}\left(f_{\alpha}\right)\right)\left(\frac{n}{b_{\alpha}} k_{\perp}+k_{\|} \frac{p_{\|}}{p_{\perp}}\right)\right. \\
& \left.+\mathcal{L}\left(f_{\alpha}\right) \frac{k_{\|}}{m_{\alpha} \gamma_{\alpha} \omega}\left[-\frac{m_{\alpha} \gamma_{\alpha} \omega}{p_{\perp}}+k_{\|} \frac{p_{\|}}{p_{\perp}}+k_{\perp} \frac{n}{b_{\alpha}}\right]\right\} \\
& +\frac{\omega}{k^{2}} \sum_{\alpha} \frac{X_{\alpha}}{n_{\alpha}} \frac{k_{\perp} \sin \psi}{m_{\alpha} \Omega_{\alpha}} \sum_{n=-\infty}^{+\infty} \int d^{3} p p_{\perp}^{2} \frac{1}{\gamma_{\alpha} D_{\alpha_{n}}} J_{n} \\
& \times\left(\frac{n}{b_{\alpha}} k_{\perp}+k_{\|} \frac{p_{\|}}{p_{\perp}}\right) \\
& \times\left\{\left[\frac{m_{\alpha} \gamma_{\alpha} \omega}{p_{\perp}}-k_{\|} \frac{p_{\|}}{p_{\perp}}-k_{\perp} \frac{n}{b_{\alpha}}\right] \frac{1}{m_{\alpha} \gamma_{\alpha} \omega} f_{\alpha}^{\prime} J_{n}\right. \\
& \left.+\frac{1}{m_{\alpha} \gamma_{\alpha} \omega} f_{\alpha}^{\prime} \frac{k_{\perp}}{k_{\perp}} J_{n}\left(\frac{n}{b_{\alpha}} k_{\perp}+k_{\|} \frac{p_{\|}}{p_{\perp}}\right)\right\} \\
& +\frac{\omega}{k^{2}} \sum_{\alpha} \frac{X_{\alpha}}{n_{\alpha}} \frac{\sin \psi}{m_{\alpha} \Omega_{\alpha}} \sum_{n=-\infty}^{+\infty} \int d^{3} p p_{\perp}^{2} \frac{1}{\gamma_{\alpha} D_{\alpha_{n}}} \\
& \times\left\{\left(\frac{\partial f_{\alpha}^{\prime}}{\partial p_{\perp}}-\frac{k_{\|}}{m_{\alpha} \gamma_{\alpha} \omega} \mathcal{L}\left(f_{\alpha}^{\prime}\right)\right)\left(k_{\perp} \frac{n}{b_{\alpha}}+k_{\|} \frac{p_{\|}}{p_{\perp}}\right)\right. \\
& \times\left[\frac{n}{b_{\alpha}} J_{n}^{2}\left(k_{\perp} \frac{n}{b_{\alpha}}+k_{\|} \frac{p_{\|}}{p_{\perp}}\right)-k_{\perp} \frac{J_{n} J_{n}^{\prime}}{b_{\alpha}}\right] \\
& +\mathcal{L}\left(f_{\alpha}^{\prime}\right) \frac{k_{\|} \sin \psi}{m_{\alpha} \gamma_{\alpha} \omega}\left[\frac{n}{b_{\alpha}} J_{n}^{2}\left(k_{\perp} \frac{n}{b_{\alpha}}+k_{\|} \frac{p_{\|}}{p_{\perp}}\right)-k_{\perp} \frac{J_{n} J_{n}^{\prime}}{b_{\alpha}}\right]
\end{aligned}
$$




$$
\left.\times\left[-\frac{m_{\alpha} \gamma_{\alpha} \omega}{p_{\perp}}+k_{\|} \frac{p_{\|}}{p_{\perp}}+k_{\perp} \frac{n}{b_{\alpha}}\right]\right\} .
$$

After some simple algebraic manipulation, we obtain the following,

$$
\begin{aligned}
& \varepsilon_{l}=1+\frac{\omega}{k^{2}} \sum_{\alpha} \frac{X_{\alpha}}{n_{\alpha}} \sum_{n=-\infty}^{+\infty} \int d^{3} p p_{\perp} \frac{1}{\gamma_{\alpha} D_{\alpha_{n}}} J_{n}^{2} \\
& \times\left(\frac{n}{b_{\alpha}} k_{\perp}+k_{\|} \frac{p_{\|}}{p_{\perp}}\right)\left[k_{\perp} \frac{n}{b_{\alpha}} \frac{\partial f_{\alpha}}{\partial p_{\perp}}+k_{\|} \frac{\partial f_{\alpha}}{\partial p_{\|}}\right] \\
& +\frac{\omega}{k^{2}} \sum_{\alpha} \frac{X_{\alpha}}{n_{\alpha}} \frac{k_{\perp}}{m_{\alpha} \Omega_{\alpha}} \sum_{n=-\infty}^{+\infty} \int d^{3} p p_{\perp} \frac{1}{\gamma_{\alpha} D_{\alpha_{n}}} \\
& +\frac{\omega}{k^{2}} \sum_{\alpha} \frac{X_{\alpha}}{n_{\alpha}} \frac{\sin \psi}{m_{\alpha} \Omega_{\alpha}} \sum_{n=-\infty}^{+\infty} \int d^{3} p p_{\perp}^{2} \frac{1}{\gamma_{\alpha} D_{\alpha_{n}}} \\
& \quad \times\left[\frac{k_{\perp}}{b_{n}^{2}}\left(k_{\|} \frac{p_{\|}}{p_{\perp}}\right) f_{\alpha}^{\prime}\right. \\
& \left.\quad \times\left[k_{\perp} \frac{n}{b_{\alpha}} \frac{\partial f_{\alpha}^{\prime}}{\partial p_{\perp}}+k_{\|} \frac{p_{\|}}{p_{\perp}}\right)-k_{\perp} \frac{\partial f_{\alpha}^{\prime}}{\partial p_{\|}}\right] .
\end{aligned}
$$

It is important to notice that none of the components $k_{i}$ labeled as coming from the magnetic fluctuations have survived up to this point.

We now write

$$
\begin{gathered}
\left(\frac{n}{b_{\alpha}} k_{\perp}+k_{\|} \frac{p_{\|}}{p_{\perp}}\right) f_{\alpha}^{\prime}=\frac{m_{\alpha} \gamma_{\alpha}}{p_{\perp}}\left(\frac{n \Omega_{\alpha}}{\gamma_{\alpha}}+\frac{k_{\|} p_{\|}}{m_{\alpha} \gamma_{\alpha}}\right) \\
=\frac{m_{\alpha} \gamma_{\alpha}}{p_{\perp}}\left(\omega-D_{\alpha_{n}}\right) .
\end{gathered}
$$

Inserting this expression into Eq. (28), and using $\sum_{n} n J_{n}^{2}=0, \sum_{n} J_{n}^{2}=1$ and $\sum_{n} n^{2} J_{n}^{2}(x)=x^{2} / 2$, and also taking into account that

$$
\int d^{3} p \frac{\partial f_{\alpha}}{\partial p_{\|}}=0, \quad \int d^{3} p p_{\perp} \frac{\partial f_{\alpha}^{\prime}}{\partial p_{\perp}}=-2 \int d^{3} p f_{\alpha}^{\prime},
$$

we obtain Eq. (13).

\section{References}

[1] V. S. Beskin, A. V. Gurevich, and Y. I. Istomin, Sov. Phys. JETP 65, 715 (1987).

[2] R. A. Caldela Filho, R. S. Schneider, and L. F. Ziebell, J. Plasma Phys. 42, 165 (1989).

[3] R. A. Caldela Filho, R. S. Schneider, and L. F. Ziebell, J. Plasma Phys. 43, 335 (1990).

[4] C. J. H. Cavalcanti, R. S. Schneider, and L. F. Ziebell, J. Plasma Phys. 52, 195 (1994).

[5] R. Gaelzer, R. S. Schneider, and L. F. Ziebell, Phys. Rev. E 51, 2407 (1995).

[6] R. Gaelzer, R. S. Schneider, and L. F. Ziebell, Phys. Rev. E 55, 5859 (1997).

[7] R. Gaelzer, L. F. Ziebell, and O. J. G. Silveira, Phys. Plasmas 6, 4533 (1999).

[8] O. J. G. Silveira, L. F. Ziebell, R. Gaelzer, and P. H. Yoon, Phys. Rev. E 65, 036407, 11p. (2002).

[9] J. B. McBride, E. Ott, J. P. Boris, and J. H. Orens, Phys. Fluids 15, 2367 (1972).

[10] D. S. Lemons and S. P. Gary, J. Geophys. Res. 83, 1625 (1978).

[11] C. S. Wu, D. Dillenburg, L. F. Ziebell, and H. P. Freund, Planet. Space Sci. 31, 499 (1983).

[12] S. T. Tsai et al., J. Plasma Phys. 32, 159 (1984).

[13] A. T. Y. Lui et al., J. Geophys. Res. 96, 11389 (1991).

[14] C. L. Chang, H. K. Wong, and C. S. Wu, Phys. Rev. Lett. 65 , 1104 (1990).

[15] P. H. Yoon and A. T. Y. Lui, Phys. Fluids B 5, 836 (1993).

[16] N. A. Krall and P. C. Liewer, Phys. Rev. A 4, 2094 (1971).

[17] R. C. Davidson and N. T. Gladd, Phys. Fluids 18, 1327 (1975).

[18] N. T. Gladd, Phys. Fluids 18, 27 (1976).

[19] R. C. Davidson, N. T. Gladd, C. S. Wu, and J. D. Huba, Phys. Fluids 20, 301 (1977).

[20] J. B. Hsia et al., Phys. Fluids 22, 1737 (1979).

[21] J. D. Huba, N. T. Gladd, and J. F. Drake, J. Geophys. Res. 86, 5881 (1981).

[22] Y. M. Zhou, H. K. Wong, C. S. Wu, and D. Winske, J. Geophys. Res. 88, 3026 (1983).

[23] P. H. Yoon, A. T. Y. Lui, and C.-L. Chang, Phys. Plasmas 1, 3033 (1994).

[24] T. H. Stix, The theory of plasma waves (AIP, New York, 1992). 